\title{
Contenido de carbono en la biomasa aérea de bosques nativos en Chile*
}

\author{
Carbon content in the above-ground biomass of evergreen forest in Chile
}

JORGE GAYOSO A., JAVIER GUERRA C.

Instituto de Manejo Forestal, Universidad Austral de Chile, Casilla 567, Valdivia, Chile.

\author{
SUMMARY
}

Carbon (C) content in stem, branches, leaves and bark from 16 tree species of the native forest in Chile was determined. The mean contents of organic $\mathrm{C}$ by species varied between $34.9 \%$ and $48.3 \%$ and the single mean was $43,7 \pm 0.16 \%$. The total $C$ content was $5,5 \pm 0.76 \%$ higher than the organic C content. Significant differences between groups of species and tree tissues were found. For all the species the stem's C contents $(44,4 \pm 0.16 \%)$ were slightly higher than those in woody branches, leaves and bark $(43,5 \pm 0.21 \%)$. The highest $\mathrm{C}$ content was for Saxegothaea conspicua with $47,8 \pm 0.38 \%$ and the lowest for Weinmannia trichosperma, 42,6 $\pm 0.54 \%$. Through analysis of variance, the study didn't show significant differences between deciduous species and evergreen species and forest type as it did for native coniferous and broadleaf.

Key words: carbon contents, above-ground biomass, temperate rainforests.

\section{RESUMEN}

Se determinó el contenido de carbono (C) en fuste, ramas, hojas y corteza para 16 especies del bosque nativo en Chile. Los contenidos promedio de C orgánico en las especies fluctuaron entre 34,9 y 48,3\%, y el promedio

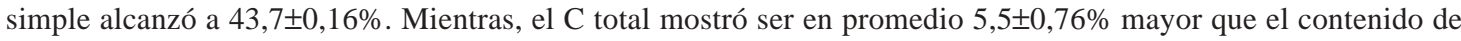
$\mathrm{C}$ orgánico. Se encontraron diferencias significativas en el contenido de $\mathrm{C}$ orgánico entre grupos de especies y entre componentes del árbol. El carbono del fuste $(44,4 \pm 0,16 \%)$, en general, para todas las especies resultó ligeramente mayor que en las ramas, hojas y corteza $(43,5 \pm 0,21 \%)$, siendo mayor la variabilidad en estos últimos. La especie con el mayor contenido de C orgánico en la biomasa del fuste resultó Saxegothaea conspicua (mañío

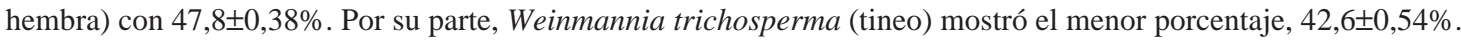
Mediante análisis de varianza y método de Bonferroni, el estudio no encontró diferencias significativas en el contenido de $\mathrm{C}$, entre las especies caducifolias y las especies siempre verdes, ni entre el grupo de especies del tipo forestal Siempreverde y del tipo Roble-Raulí-Coigüe. Por el contrario, se encontraron diferencias en el contenido de $\mathrm{C}$ entre las coníferas nativas y las especies latifoliadas.

Palabras clave: contenido de carbono, biomasa aérea, bosques siempre verdes.

\section{INTRODUCCION}

El dióxido de carbono presente en la atmósfera es absorbido por las plantas, a través del proceso de fotosíntesis. Por este medio, las plantas con- vierten la energía de la luz solar en energía química aprovechable para los organismos vivos. Así, los bosques almacenan grandes cantidades de carbono (C) en la vegetación y el suelo, e intercambian $\mathrm{C}$ con la atmósfera a través de la fotosíntesis y la respiración.

* La presente investigación fue desarrollada como parte del proyecto "Medición de la capacidad de captura de carbono en bosques de Chile y promoción en el mercado mundial", financiado con aporte del Fondo de Desarrollo Científico y Tecnológico de Chile (FONDEF). 
En general, se acepta que el contenido de C corresponde al $50 \%$ de la biomasa $(1,2)$. Sin embargo, diferentes estudios denotan la variabilidad del contenido de $\mathrm{C}$ según especie y tejido del árbol $(3,4)$. El IPCC (2) señala también que el rango más citado para el contenido de $\mathrm{C}$ en la biomasa es 43 a 58\%. Cubero y Rojas (5) señalan que el contenido de $\mathrm{C}$ en la biomasa arbórea se ve influenciado por la calidad del sitio y edad de las plantaciones, obteniendo contenidos de $\mathrm{C}$ entre 32 y $40 \%$ para plantaciones de Gmelina arborea, 32 a $36 \%$ para Tectona grandis y 33 a $36 \%$ para Bombacopsis quinata. Por otra parte, también se han encontrado diferencias en el contenido de $\mathrm{C}$, según el método empleado en su determinación (6).

El conocimiento del contenido de carbono en la biomasa es de utilidad para la determinación del balance nacional de Gases de Efecto Invernadero, la construcción de los indicadores nacionales respectivos del Proceso de Montreal y el levantamiento de líneas de base para futuros proyectos negociables en el mercado del C.

\section{MATERIAL Y METODOS}

El estudio comprende 16 especies nativas de los tipos forestales Siempreverde y Roble-RaulíCoigüe ${ }^{1}$, cuadro 1 . Las muestras se colectaron en 53 parcelas de 7 áreas forestales considerando una amplia distribución latitudinal ( $38^{\circ} \mathrm{S}$ a $\left.40^{\circ} 30^{\prime} \mathrm{S}\right)$, longitudinal $\left(72^{\circ} \mathrm{W}\right.$ a $\left.73^{\circ} 30^{\prime} \mathrm{W}\right)$ y altitudinal $(200$ a $880 \mathrm{~m}$ ) y con un rango de precipitaciones variable entre 1.500 y $4.000 \mathrm{~mm}$ anuales.

El muestreo en los tipos forestales seleccionados se ajustó a la disponibilidad de los predios aportados por las empresas forestales asociadas al estudio, a partir de los cuales se ubicaron los sitios más representativos del tipo forestal. En cada área de muestreo se establecieron cuatro parcelas de $500 \mathrm{~m}^{2}$ cada una, en parejas distribuidas al azar, separadas a una distancia de 60 metros. Para la correcta ubicación de las parcelas, en terreno se utilizó material cartográfico y equipos de sistema de posicionamiento global (GPS). A partir de los inventarios de las parcelas, se seleccionaron los árboles muestra. El método consideró la tabla

\section{CUADRO 1}

Especies nativas estudiadas Studied Native Species

\begin{tabular}{|llll|}
\hline TIPO FORESTAL & Nombre Común & Nombre científico & Abreviatura \\
\hline \multirow{6}{*}{ Siempreverde } & Luma & Amomyrtus luma & AL \\
& Trevo & Dasyphyllum diacanthoides & DD \\
& Canelo & Drimys winteri & DW \\
& Ulmo & Eucryphia cordifolia & EC \\
& Avellano & Gevuina avellana & GA \\
& Tepa & Laureliopsis philippiana & LP \\
& Coigüe común & Nothofagus dombeyi & ND \\
Coigüe de Chiloé & Nothofagus nitida & NN \\
& Mañío macho & Podocarpus nubigena & PN \\
& Mañío hembra & Saxegothaea conspicua & SC \\
& Tineo & Weinmannia trichosperma & WT \\
\hline \multirow{6}{*}{ Roble-Raulí-Coigüe } & Olivillo & Aextoxicon punctatum & AP \\
& Avellano & Gevuina avellana & GA \\
& Laurel & Laurelia sempervirens & LS \\
Raulí & Nothofagus alpina & NA \\
& Roble & Nothofagus obliqua & NO \\
& Lingue & Persea lingue & PL \\
\hline
\end{tabular}

1 Tipificación de acuerdo a Donoso (9). 
de frecuencia, según clase diamétrica para determinar el número de individuos a muestrear por especie en cada sitio, pero asegurando, además, al menos un individuo de cada especie por cada clase diamétrica con frecuencia de al menos tres individuos (7).

Los suelos mayoritariamente de origen volcánico (Andisoles) se han desarrollado de cenizas modernas depositadas sobre rocas andesíticas para los sitios próximos a la Cordillera Andina y de cenizas antiguas sobre el complejo metamórfico para los casos de la Cordillera de la Costa. Son suelos con buen drenaje, moderadamente profundos a profundos, y de textura franco a franco limosa en el sector andino y franco limoso a arcillo limosa hacia la costa (8).

Se analizaron 2.034 muestras provenientes de la madera del fuste, corteza, ramas y hojas de 409 árboles. Las muestras fueron molidas y secadas en estufa a $103 \pm 2^{\circ} \mathrm{C}$, para luego determinar el contenido de carbono orgánico mediante el método de Walkey y Black descrito por TESAM y CONAMA (10) y que consiste en la oxidación con una solución de dicromato de potasio en un medio sulfúrico. Según la cantidad de reactivo utilizado en la reacción, se calculó la equivalencia de carbono presente en la muestra; la determinación se realizó colorimétricamente midiendo la intensidad del color producido por los iones de cromo. Cabe señalar que este método sólo evalúa la fracción orgánica de carbono en el tejido vegetal. Además, para establecer las diferencias y relaciones que permitan la estimación del contenido de $\mathrm{C}$ total a partir del $\mathrm{C}$ orgánico, se realizaron 44 ensayos sobre muestras dobles para los diferentes tejidos del árbol, utilizando el método colorimétrico para establecer carbono orgánico y de combustión o calorimetría para obtener carbono total (5). Mediante análisis de varianza, test de rangos múltiples y análisis de conglomerados, se establecieron las diferencias significativas entre las medias del contenido de $\mathrm{C}$ de las especies, componentes del árbol y grupos de especies.

\section{RESULTADOS Y DISCUSION}

El contenido de carbono orgánico expresado como porcentaje de la biomasa seca en los componentes del árbol y en las especies estudiadas varió entre $34,86 \%$ y $48,31 \%$, con una media sim- ple de $43,68 \pm 0,16 \%$ (cuadro 2). El estudio muestra variaciones significativas según especie y tejido del árbol analizado al 95\% de confianza.

La especie con menor contenido de $\mathrm{C}$ en la madera de los fustes resultó Weinmannia trichosperma $(42,60 \%)$ y la mayor Saxegothaea conspiсиа $(47,78 \%)$. Las mayores variaciones del contenido de $\mathrm{C}$ las presentan las hojas para el conjunto de especies, y dentro de cada especie, las ramas. Además, la variación entre especies resultó mayor que la variación entre tejidos y no se encontró una relación entre diámetro del árbol y el contenido de $\mathrm{C}$ de la madera. Igualmente, debido a la variabilidad interespecífica, no se lograron diferencias entre el conglomerado de especies del bosque Roble-Raulí-Coigüe y el bosque Siempreverde, ni entre las especies caducifolias y siempre verdes. La variabilidad del contenido de $\mathrm{C}$ ha sido referida por algunos autores: Birdsey (11), citado por McNulty (12), señala para la fracción de carbono valores de 50 a $53 \%$, dependiendo del tipo de bosque en EE.UU, y Kurbanov (13), en cambio, entrega un amplio rango entre 47 y $58 \%$ para Pinus silvestris. Otro estudio sobre 19 especies en Australia muestra variaciones del contenido de C, obtenidas con espectrómetro de masas, entre 45 y $55 \%$, según especie y tejido (4). Francis (3), evaluando bosques secundarios en estado de latizal en Puerto Rico, reporta para 20 especies valores de contenido de C entre 45 y $55,4 \%$, obtenido mediante combustión.

Para calcular el contenido de $\mathrm{C}$ promedio ponderado por árbol, es necesario conocer la distribución de la biomasa en los diferentes componentes del mismo ${ }^{2}$, siendo el fuste el que aporta la mayor cantidad de biomasa y el cual lleva al promedio ponderado por sobre el promedio simple. La variación del contenido de carbono en los tejidos del árbol ha sido escasamente documentada en Chile. Uno de los estudios más extensos realizados en Australia (4) señala que el contenido de carbono dependerá de la proporción de compuestos como la lignina y minerales inorgánicos. Según esto, como los contenidos de

\footnotetext{
2 Esta distribución se asocia a las características de la especie, estado de desarrollo, posición sociológica y atributos del rodal (15). El mismo estudio señala los siguientes valores promedio para similar conjunto de especies nativas: fuste $71,35 \%$, ramas $13,44 \%$, hojas $4,28 \%$ y corteza $10,92 \%$.
} 
BOSQUE 26(2): 33-38, 2005

Contenido de carbono en la biomasa aérea de bosques nativos en Chile

\section{CUADRO 2}

Contenido de carbono en especies nativas (\%). Tamaño de la muestra (N), desviación estándar (SD) y media. Carbon content in native species (\%). Sample size (N), Standard deviation (SD) and mean.

\begin{tabular}{|c|c|c|c|c|c|c|c|c|c|c|c|c|}
\hline \multirow[b]{3}{*}{ Especie } & \multicolumn{12}{|c|}{ Tejido } \\
\hline & \multicolumn{3}{|c|}{ Fuste } & \multicolumn{3}{|c|}{ Hojas } & \multicolumn{3}{|c|}{ Ramas } & \multicolumn{3}{|c|}{ Corteza } \\
\hline & $\mathrm{N}$ & SD & Media & $\mathrm{N}$ & SD & Media & $\mathrm{N}$ & SD & Media & $\mathrm{N}$ & SD & Media \\
\hline $\mathrm{AL}$ & 21 & 1,46 & 43,32 & 18 & 3,06 & 41,80 & 18 & 3,72 & 42,23 & 21 & 2,90 & 36,90 \\
\hline AP & 11 & 1,87 & 43,33 & 9 & 4,80 & 40,18 & 9 & 4,74 & 40,87 & 7 & 2,01 & 36,73 \\
\hline DD & 12 & 1,09 & 43,60 & 9 & 2,57 & 34,86 & 9 & 2,79 & 39,39 & 12 & 1,27 & 41,66 \\
\hline DW & 92 & 1,61 & 44,74 & 67 & 4,00 & 44,47 & 66 & 3,77 & 48,31 & 92 & 3,94 & 47,37 \\
\hline $\mathrm{EC}$ & 55 & 1,82 & 43,56 & 42 & 2,69 & 45,00 & 42 & 2,89 & 42,18 & 46 & 2,63 & 44,32 \\
\hline GA & 27 & 0,90 & 43,35 & 18 & 2,16 & 38,21 & 18 & 1,92 & 40,77 & 22 & 1,96 & 41,50 \\
\hline LP & 63 & 1,58 & 45,00 & 51 & 3,54 & 42,51 & 48 & 1,82 & 42,93 & 62 & 1,97 & 43,37 \\
\hline LS & 11 & 0,97 & 44,45 & 3 & 0,90 & 40,36 & 6 & 1,24 & 42,62 & 5 & 2,55 & 43,20 \\
\hline NA & 97 & 1,85 & 44,39 & 49 & 2,74 & 43,60 & 48 & 1,80 & 41,63 & 51 & 3,29 & 45,15 \\
\hline ND & 24 & 1,86 & 43,07 & 18 & 2,15 & 40,78 & 18 & 2,14 & 39,93 & 24 & 2,12 & 41,55 \\
\hline NN & 35 & 0,60 & 44,43 & 27 & 2,82 & 47,69 & 27 & 2,33 & 44,31 & 36 & 1,56 & 44,76 \\
\hline $\mathrm{NO}$ & 54 & 2,46 & 43,53 & 33 & 3,69 & 41,56 & 33 & 2,62 & 39,56 & 34 & 4,16 & 39,82 \\
\hline PL & 20 & 0,73 & 43,51 & 14 & 1,44 & 45,12 & 12 & 2,51 & 43,18 & 17 & 1,32 & 44,03 \\
\hline PN & 40 & 1,28 & 47,31 & 30 & 2,94 & 45,09 & 30 & 2,21 & 43,44 & 39 & 2,52 & 40,09 \\
\hline $\mathrm{SC}$ & 27 & 0,96 & 47,78 & 21 & 1,94 & 48,15 & 21 & 1,57 & 44,58 & 28 & 1,15 & 44,21 \\
\hline WT & 40 & 1,68 & 42,60 & 30 & 2,27 & 41,11 & 30 & 4,08 & 43,69 & 35 & 3,26 & 44,31 \\
\hline Total & 629 & 2,06 & 44,38 & 439 & 4,02 & 43,35 & 435 & 3,78 & 43,17 & 531 & 3,95 & 43,52 \\
\hline
\end{tabular}

lignina y minerales difieren entre los distintos tejidos del árbol, es esperable que los diversos componentes del árbol presenten distintos contenidos de carbono.

Mediante análisis de varianza, se rechaza la hipótesis nula de igualdad de los contenidos de carbono entre especies y tejidos. Se confirma la existencia de diferencias significativas entre los contenidos de carbono promedio de las diferentes especies nativas $(\mathrm{F}=45,17 ; \mathrm{P}$ valor $=0,0000) \mathrm{y}$ entre sus tejidos $(\mathrm{F}=19,33$; $\mathrm{P}$ valor $=0,0000)$

Estudiados los contenidos de carbono entre los tejidos del árbol, se establecieron dos agrupamientos: uno donde se asocian los tejidos de ramashojas-corteza $(43,50 \pm 0,21 \%)$ y un segundo grupo donde sólo se separa el fuste $(44,38 \pm 0,16 \%)$. Para el caso de la madera del fuste no se hizo diferenciación entre madera de albura y duramen.

Este mayor contenido de carbono en la madera del fuste en relación a los restantes tejidos puede deberse a un menor contenido de minerales y mayor contenido de lignina que en hojas y ramas. Sin embargo, estudios en Australia sobre Eucalyp- tus sp. y Pinus radiata mostraron la relación contraria, es decir, los contenidos de carbono fueron mayores en hojas verdes que en ramas leñosas, mientras el duramen presentó mayor contenido de carbono que la madera de albura (4). Por otra parte, Segura (6) no encontró diferencias entre los diferentes tejidos y secciones de la especie Quercus costaricensis.

Mediante análisis de cluster, el contenido de carbono de los fustes se agrupó en dos conglomerados: uno que contienen las coníferas nativas ( $\mathrm{Po}$ docarpus nubigena y Saxegothaea conspicua) con un promedio de $47,50 \pm 0,29 \%$ y otro que contiene el resto de especies latifoliadas $(44,01 \pm 0,15 \%)$ (figura 1). Similar tendencia es señalada por Lopera y Gutiérrez (14), quienes señalan un rango entre 50 y $53 \%$ para el contenido de $\mathrm{C}$ de la madera de coníferas y 47 a $50 \%$ para especies de hoja ancha. Igualmente, Birdsey (11), citado por Slijepcevic (1), respecto de los porcentajes promedio de contenido de $\mathrm{C}$ total, señala un valor de $52,1 \%$ para coníferas y $49,1 \%$ para madera de árboles de hoja ancha. 
BOSQUE 26(2): 33-38, 2005

Contenido de carbono en la biomasa aérea de bosques nativos en Chile

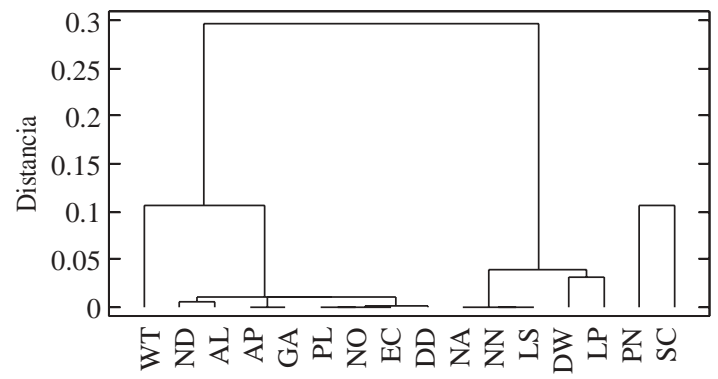

Figura 1. Dendrograma del contenido de carbono en fustes por especie.

Carbon content dendrogram in stems by species.

Dentro de las especies latifoliadas, se identificaron, a su vez, dos grupos: el primero conteniendo las especies Nothofagus alpina, Nothofagus nitida, Laurelia sempervivens, Drimys winteri y Laureliopsis philippiana con 44,63 $\pm 0,18 \%$ y el segundo, el resto de las especies con 43,31 $\pm 0,21 \%$.

Manteniendo la agrupación anterior, se obtuvo que el contenido de carbono en los tejidos de ramas, hojas y corteza corresponde a $43,81 \pm 0,49 \%$ para las coníferas nativas y $43,30 \pm 0,22 \%$ para las demás nativas latifoliadas. No obstante, la agrupación para estos tejidos no es correspondiente con el agrupamiento por fuste, y por su menor peso relativo se sugiere agruparlas, en cuyo caso el contenido de carbono es igual a 43,36 $\pm 0,20 \%$.

El contenido de carbono de la biomasa vegetal, obtenido con el método colorimétrico, resultó en todos los casos inferior al valor por defecto sugerido por el Panel Intergubernamental de Cambio Climático (2) e inferior a valores reportados por otros autores $(3,4,11)$. Algunas excepciones son la relación utilizada por Ortiz (16) para bosques secundarios de Costa Rica, quien lo fija en $45 \%$, monto que se acerca a los valores determinados en este estudio; también Segura (6) con 45\% para madera de Quercus sp., y Santa Regina y Tarazona (17), quienes determinan en $45,7 \pm 1,1 \%$ el contenido de carbono en hojas de la especie Fagus sylvatica.

Este menor valor se debería a que el método utilizado evalúa sólo la fracción orgánica del material analizado, mientras que otros métodos evalúan, además, la fracción inorgánica. La elección del método colorimétrico se debió exclusivamente a restricciones presupuestarias, ya que una determinación de carbono total por métodos como el de calorimetría llega a ser 12 a 15 veces superior al costo del ensayo colorimétrico.
Analizadas las diferencias, se rechazó la hipótesis nula que los contenidos de carbono obtenidos con ambos métodos eran iguales, siendo en todos los casos el carbono total superior al contenido de carbono orgánico $(\mathrm{F}=118,81 ; \mathrm{P}$ valor $=$ 0,0000). Además, estas diferencias resultaron mayores para las hojas y menores para la madera del fuste; sin embargo, no se encontraron diferencias significativas entre tejidos (cuadro 3 ).

\section{CUADRO 3}

Diferencias entre carbono orgánico y total por componente del árbol

Differences between organic and total carbon content by tree tissue

\begin{tabular}{|llc|}
\hline Tejido & $\mathrm{N}$ & Diferencia (\%) \\
\hline Fuste & 12 & $4,61 \pm 1,18$ \\
Hojas & 10 & $6,33 \pm 2,32$ \\
Ramas & 10 & $5,35 \pm 2,05$ \\
Corteza & 12 & $5,86 \pm 1,19$ \\
Total & 44 & $5,51 \pm 0,76$ \\
\hline
\end{tabular}

Lo anterior permite estimar el contenido total de carbono para las especies nativas estudiadas a partir del contenido de carbono orgánico, agregando una diferencia única de $5,51 \pm 0,76 \%$ a los valores presentados en el cuadro 2 .

En consecuencia, se puede decir que el contenido de carbono total para la madera de fustes varía entre $53,01 \pm 1,05 \%$ para las coníferas nativas y $49,52 \pm 0,91 \%$ para las restantes 14 especies estudiadas. Por otra parte, si se acepta que el fuste representa el 71,35\% de la biomasa del árbol, y se consideran todas las especies estudiadas como un conjunto, el contenido de carbono total de la biomasa aérea (hojas, ramas, corteza y fuste) alcanza un valor promedio de $49,64 \pm 0,93 \%$.

\section{CONCLUSIONES}

Se encontraron variaciones significativas del contenido de carbono entre las especies nativas estudiadas, las que en el tejido de los fustes superaron los cinco puntos porcentuales. Igualmente se encontraron diferencias significativas al $95 \%$ entre el carbono de la madera de los fustes y la de tejidos de hojas, ramas y corteza. 
26(2):33-38, 2005

Contenido de carbono en la biomasa aérea de bosques nativos en Chile

Para efectos de la cuantificación del carbono en proyectos transables de captura de carbono, debiera preferirse el empleo de contenidos de carbono específicos, para lo cual es necesario contar con información diferenciada por especie y componente del árbol. No obstante, si se requiere una cifra única del contenido de carbono de la biomasa aérea del tipo forestal siempreverde, puede emplearse el valor $49,64 \pm 0,93 \%$.

Las diferencias entre carbono orgánico y total presentan en este estudio aún una fuerte variación, por lo cual se sugiere aumentar el número de ensayos y estudiar la posibilidad de obtener valores más precisos por conglomerados de tejidos o especies.

\section{BIBLIOGRAFIA}

(1) SLIJEPCEVIC, A. Loss of carbon during controlled regeneration burns in Eucalyptus obliqua forest. Tasforests, 2001, vol. 13, $\mathrm{N}^{\circ} 2$, p. 281-290.

(2) INTERGOVERNMENTAL PANEL ON CLIMATE CHANGE. Chapter 5: Land Use Change \& Forestry. Greenhouse Gas Inventory Reference Manual. IPCC Guidelines for National Greenhouse Gas Inventories, Revised Version. London, 1996, vol. 3, 57 p.

(3) FRANCIS, J. Estimating Biomass and Carbon Content of Saplings in Puerto Rican Secondary Forests. Caribbean Journal of Science, 2000, vol. 36, $\mathrm{N}^{\circ} 3-4$, p. 346-350.

(4) GIFFORD, R. Carbon contents of above-ground tissues of forest and woodland trees. Canberra: Australian Greenhouse Office, National Carbon Accounting System, 2000 Technical Report $\mathrm{N}^{\mathrm{o}} 22.17 \mathrm{p}$.

(5) CUBERO, J., S. ROJAS. Fijación de carbono en plantaciones de Gmelina arborea, Tectona grandis y Bombacopsis quinata en los cantones de Hojancha y Nicoya Guanacaste, Costa Rica. Heredia (Costa Rica): Tes. Lic. Cs. Forestales Universidad Nacional, 1999. 93 p.

(6) SEGURA, M. Almacenamiento y fijación de carbono en Quercus costaricensis, en un bosque de altura en la
Cordillera de Talamanca, Costa Rica. Heredia (Costa Rica): Tes. Lic. Cs. Forestales. Universidad Nacional, 1999. $127 \mathrm{p}$

(7) SCHLEGEL, B., J. GAYOso, J. GUERRA. Métodos de medición de biomasa forestal. Proyecto Medición de la Capacidad de Captura de Carbono en Bosques de Chile y Promoción en el Mercado Mundial de Carbono. Universidad Austral de Chile, 2000. 18 p.

(8) INSTITUTO DE INVESTIGACIONES AGROPECUARIAS. Suelos volcánicos de Chile. Santiago (Chile): Ministerio de Agricultura, 1995. $723 \mathrm{p}$.

(9) DONOSO, C. Tipos forestales de los Bosques Nativos de Chile. Investigación y desarrollo forestal (CONAF/ PNUD/FAO). Santiago (Chile): Publicación FAO, Documento de Trabajo $\mathrm{N}^{\circ} 38,1981.83 \mathrm{p}$.

(10) TESAM S.A., CONAMA. Metodologías para la caracterización de la calidad ambiental. Santiago (Chile): Comisión Nacional del Medio Ambiente, 1996. 242 p.

(11) BIRDSEY, R. Carbon Storage and Accumulation in the United States Forest Ecosystems. USDA, Forest Service General Technical Report WO-59. Washington, DC, 1992. $51 \mathrm{p}$.

(12) McNULTY, S. Hurricane impacts on US forest carbon sequestration. Environmental Pollution, 2002, Volume 116, Supplement 1, p. 17-24.

(13) KURBANOV, E. Carbon Pine Forest Ecosystems of Middle Zavolgie, Russia. European Forest Institute, 2000, International Report $\mathrm{N}^{\circ} 2.68 \mathrm{p}$.

(14) LOPERA, G., V. GUTIERREZ. Viabilidad técnica y económica de la utilización de plantaciones de Pinus patula como sumideros de $\mathrm{CO}_{2}$. Medellín: Tes. Ing. Forestal. Universidad Nacional de Colombia, 2000. $146 \mathrm{p}$.

(15) GAYOSO, J., J. GUERRA, D. ALARCON. Contenido de carbono y funciones de biomasa en especies nativas y exóticas. Valdivia (Chile): Universidad Austral de Chile, Proyecto medición de la capacidad de captura de carbono en bosques de Chile y promoción en el mercado mundial. Informe Final, Documento $\mathrm{N}^{\circ} 1$, 2002. 53 p.

(16) ORTIZ, E. Costa Rica secondary forest: an economic option for joint implementation initiatives to reduce atmospheric $\mathrm{CO}_{2}$. Draft paper presented for inclusion in the Beijer Seminar in Punta Leona. Costa Rica, 1997. $19 \mathrm{p}$.

(17) SANTA REGINA, I., T. TARAZONA. Nutrient cycling in a natural beech forest and adjacent planted pine in northern Spain. Forestry, 2001, vol. 74 No 1, p. 11-28. 